\title{
II. REVIEWS.
}

Abbe, C. The Mechanics of the Earth's Atmosphere. A Collection of Translations (third), E. W. BRown, xx, 435.

Abhandlungen zur Geschichte der mathematischen Wissenschaften, 18. Heft (Heiberg, Müller, Lindt), D. E. SMITH, xII, 314.

Abraham, M. Theorie der Elektrizität. Erster Band: Einführung in die Maxwellsche Theorie der Elektrizität, von A. Föppl, (zweite Auflage), E. B. WILson, xI, 383; (vierte Auflage), J. B. SHAW, XIX, 483.

- Theorie der Elektrizität. Zweiter Band: Elektromagnetische Theorie der Strahlung, E. B. Wirson, (erste Auflage), xIv, 230; (zweite Auflage), XvI, 545 .

Adhémar, R. d'. Exercices et Lecons d'Analyse, M. Bôcher, xvi, 87.

Allen, J. See Tanner, J. H.

Amodeo, F. Complementi di Analisi algebrica elementare, C. L. E. MOORE, XVII, 261.

Andoyer, H. Cours d'Astronomie. Première Partie: Astronomie théorique, K. LAVES, XIII, 518; (deuxième édition), XviII, 517.

- Cours d'Astronomie. Seconde Partie: Astronomie pratique, W. R. LONGLEY, XV, 467.

- Nouvelles Tables trigonométriques fondamentales, E. W. BRown, Xvirr, 365.

André, D. Notations mathématiques, G. A. MiluER, xvIr, 43.

Andrews, W. S. Magic Squares and Cubes, G. A. MrLuER, Xvi, 85.

Annuaire astronomique pour 1905 (Royal Belgian Observatory), E. W. BRown, XI, 164.

Annuaire du Bureau des Longitudes, E. W. Brown, xI, 445; XII, 316; xIv, 338; xv, 364; xvI, 328; xvII, 376; xvIII, 368; xIX, 428; xx, 434 .

Appell, P., and Dautheville, S. Précis de Mécanique rationelle, J. B. SHAW, xvIII, 311.

Arendt, G. Dirichlet's Vorlesungen über die Lehre von den einfachen und mehrfachen bestimmten Integralen, V. SNYDER, xI, 371.

Ariès, E. La Statique chimique basée sur les deux Principes fondamentaux de la Thermodynamique, E. B. WiLson, xIII, 407.

Arnoux, G. Arithmétique graphique. Introduction a l'Etude des Fonctions arithmétiques, W. H. BUSSEY, XIII, 402.

- Arithmétique graphique. Les Espaces arithmétiques, leurs Transformations, W. H. Bussey, Xv, 255.

- Essai de Géométrie analytique modulaire à deux Dimensions, L. E. Dickson, $\mathrm{xx}, 96$.

Aschkinass, E. See Fleming, J. A.

Auerbach, F. Taschenbuch für Mathematiker und Physiker, 1909, J. B. SHAW, Xvi, 321.

Auerbach, F., and Rothe, R. Taschenbuch für Mathematiker und Physiker, 1911, E. W. Ponzer, XIX, 21; 1913, G. A. Mrluer, Xx, 201.

Bachelier, L. Calcul des Probabilités, H. L. RIEtz, xx, 268. 
Bachmann, P. Zahlentheorie. Fünfter Teil: Allgemeine Arithmetik der Zahlkörper, L. E. Dickson, xIII, 348.

- Grundlehren der neueren Zahlentheorie, J. W. Young, xv, 463.

Niedere Zahlentheorie, zweiter Teil, L. E. Dickson, XVII, 255.

Baillaud, B. See Hermite, C.

Baire, R. Leçons sur les Fonctions discontinues (redigées par A. Denjoy), W. D. A. WeSTFALL, XIII, 512.

— Leçons sur les Théories générales de l'Analyse, E. R. HmDrick, Xvi, 239.

Baker, H. F. An Introduction to the Theory of Multiply Periodic Functions, J. I. HuTchinson, xvi, 516. See Sylvester, J. J.

Ball, W. W. R. Histoire des Mathématiques (traduite par L. Freund), Tome premier, D. E. SmItr, XII, 309; Tome deuxième, D. E. SMItH, $\mathrm{xv}, 244$.

- Récréations mathématiques et Problèmes des Temps anciens et modernes (deuxième édition française, traduite par J. Fitz-Patrick), D. E. SMITH, XVI, 36.

Barbarin, P. See Halsted, G. B.

Barbieri, A. See Bortolotti, E.

Bartlett, G. M. See Church, A. E.

Bauer, G. Vorlesungen über Algebra (zweite Auflage), A. Dresden, XVII, 428.

Beck, H. See Bôcher, M.

Becker, G. F., and Van Orstrand, C. E. Hyperbolic Functions, J. B. SHAW, XVII, 319.

Beltrami, E. Opere matematiche, Tome I-II, E. Study, xvi, 147.

Bennecke, F. Eine konforme Abbildung als zweidimensionale Logarithmentafel zur Rechnung mit komplexen Zahlen, E. J. Townsend, xvI, 214.

Bericht der Unterrichts-Kommission der Gesellschaft deutscher Naturforscher und Aertzte über ihre bisherige Tätigkeit, J. W. A. Young, XII, 347.

Bertini, E. Introduzione alla Geometria proiettiva degli Iperspazi, C. H. SisAM, XIV, 450.

Beutel, E. Algebraische Kurven, zweiter Teil: Theorie und Kurven dritter und vierter Ordnung, H. S. WHITE, XIX, 417.

Bianchi, L. Vorlesungen über Differentialgeometrie (Deutsche Uebersetzung von M. Lukat, zweite Auflage), L. P. EIsenHarT, xvIII, 411.

Biermann, P. Vorlesungen über mathematische Näherungsmethoden, J. W. YodNG, XIII, 405.

Blaschke, E. Vorlesungen über mathematische Statistik (Die Lehre von den statistischen Masszahlen), H. L. RIETz, xv, 142.

Blaschke, W. See Study, E.

Bôcher, M. An Introduction to the Study of Integral Equations, G. A. BLIss, xvi, 207.

- Introduction to Higher Algebra. Einführung in die höhere Algebra (Deutsch von H. Beck), A. RaNUM, xvi, 521.

Boehm, K. Elliptische Funktionen, L. W. Dowling, erster Teil, xvII, 202; zweiter Teil, xIx, 146.

Bois, G. P. Tafeln unbestimmter Integrale, E. L. DodD, xv, 364. 
Bolza, O. Lectures on the Calculus of Variations, E. R. HedRICK, xII, 80, 172.

Vorlesungen über Variationsrechnung, T. H. GRoNwALL, xx, 422.

Bonola, R. La Geometria non-euclidea, Espositione storico-critica del suo Sviluppo. Die nichteuklidische Geometrie, historisch-kritische Darstellung ihrer Entwicklung (Deutsche Ausgabe von H. Liebmann), A. RANUM, XVI, 490.

- Non-Euclidean Geometry (English translation by H. S. Carslaw), A. RANUM, XIX, 22.

Bopp, K. Die Kegelschnitte des Gregorius a St. Vincentio in vergleichender Bearbeitung, F. CAJORI, xIV, 337.

Borel, E. Géométrie, Premier et Second Cycle, C. L. E. Moone, XIr, 361.

- Lecons sur les Fonctions de Variables réeles et les Développements en Séries de Polynomes (redigées par M. Fréchet), J. W. YounG, XII, 399.

- Die Elemente der Mathematik (Deutsche Ausgabe von P. Stäckel), erster Band: Arithmetik und Algebra, F. CAJORI, xVI, 89; zweiter Band: Geometrie, C. H. Sisam, Xvi, 540.

Théorie de la Croissance, R. D. Carmichael, xvir, 365.

Bortolotti, E. Lezioni sul Calcolo degli Infinitesimi (raccolte dal Dr. A. Barbieri), O. Veblen, XII, 503.

Boulanger, A. Hydraulique générale, J. B. SHAw, xvi, 382.

Bourget, H. See Hermite, C.

Boutroux, P. Lecons sur les Fonctions définies par les Equations différentielles du premier Ordre, C. L. E. Moore, Xvi, 318.

Breckenridge, W. E., Mersereau, S. F., and Moore, C. F. Shop Problems in Mathematics, C. F. CraIG, XvII, 376.

Brenke, W. C. A Text-book on Advanced Algebra and Trigonometry, A. DResden, XVII, 540.

Brill, A. Das Relativitätsprinzip. Eine Einführung in die Theorie, E. B. WILson, XIX, 321.

Brillouin, M. Propagation de l'Electricité: Histoire et Théorie, S. J. BARNETT, XII, 141.

Brioschi, F. Opere matematiche, Vols. I-III, H. S. WHITE, XII, 408.

Broggi, U. Traité des Assurances de la Vie (French translation by S. Lattès), G. H. LING, xIv, 296.

Bromwich, T. J. I'a. Quadratic Forms and their Classification by Means of Invariant Factors, M. BÖCHER, XIV, 194.

Brown, H. G. See Fisher, I.

Bruns, H. Wahrscheinlichkeitsrechnung und Kollektivmasslehre, H. L. RIETZ, XIV, 31.

— Das Gruppenschema für zufällige Ereignisse, H. L. RIETZ, XVIr, 316.

Bryan, G. H. Thermodynamics: An Introductory Treatise dealing mainly with First Principles and their Direct Applications, E. B. Wilson, XIV, 139.

Bucherer, A. H. Elemente der Vektor-Analysis (zweite Auflage), E. B. WILSON, XII, 316.

Burali-Forti, C., and Marcolongo, R. Elementi di Calcolo vettoriale con numerose Applicazioni, E. B. Wilson, xvI, 415.

- Omografie vettoriali con Applicazioni, E. B. WILson, xvi, 415.

- Calcul vectoriel (traduit par S. Lattès), E. B. WILson, xvII, 256.

Burkhardt, H. Elliptische Funktionen (zweite Auflage), J. Pierpont, xIV, 194. 
- Vorlesungen über die Elemente der Differential- und Integralrechnungen und ihre Anwendungen zur Beschreibung von Naturerscheinungen, L. W. Dowling, xvi, 79.

Bützberger, F. Über Bizentrische Polygone, Steinersche Kreis- und Kugelreihen und die Erfindung der Inversion, A. EmCH, Xx, 412.

Cadenat, A. See Russell, B.

Cajori, F. Theory of Equations, L. E. Dickson, xI, 163.

Cahen, A. See Vivanti, G.

Campbell, D. F. Differential Equations, C. R. MacInnes, xIII, 513.

Cantor, M. Vorlesungen über Geschichte der Mathematik, vierter Band, D. E. SмIтH, Xv, 352.

Carslaw, H. S. Introduction to the Theory of Fourier's Series and Integrals and the Mathematical Theory of the Conduction of Heat, J. E. Wright, Xv, 196.

- An Introduction to the Infinitesimal Calculus. Notes for the Use of Science and Engineering Students (second edition), A. M. KENYoN, $\mathrm{xx}, 204$. See Bonola, $\mathrm{R}$.

Carus, P. The Foundations of Mathematics, F. W. Owens, xvI, 541.

Carvallo, E. Calcul des Probabilités et ses Applications, A. C. LUNN, XIX, 535 .

Cattell, J. McK. American Men of Science. A Biographical Directory, G. A. MilleR, XIII, 33.

Cesàro, E. Elementares Lehrbuch der algebraischen Analysis und der Infinitesimalrechnung (Deutsch von G. Kowalewski), C. L. E. MoorE, XII, 123.

Chicago Symposium on Mathematics for Engineering Students, H. W. TYLeR, Xv, 450 .

Christoffel, E. B. Gesammelte mathematische Abhandlungen (unter Mitwirkung von A. Krazer und G. Faber, herausgegeben von L. Maurer), L. P. EISENHART, Xx, 476.

Church, A. E., and Bartlett, G. M. Elements of Descriptive Geometry, V. SNYDER, XX, 253.

Chwolson, O. D. Traité de Physique (traduit par E. Davaux), Vols. I-II, E. B. WILson, xviII, 497.

Ciani, E. Lezioni di Geometria proiettiva ed analitica, E. B. Cowlex, $\mathrm{xx}, 419$.

Clark, J. J. The Slide Rule, F. CAJori, xvi, 327.

Classen, J. Zwölf Vorlesungen über die Natur des Lichtes, E. B. WiLson, XII, 265.

Clebsch, A. and Lindemann, F. Vorlesungen über Geometrie (zweite Auflage), erster Band, erster Teil, erste und zweite Lieferung, J. LIPKA, $\mathrm{xx}, 381$.

Coffin, J. G. Vector Analysis, H. B. Phillips, xvir, 100; second edition, J. B. SHAw, XIX, 18.

Cohen, A. Differential Equations, C. R. MacInnes, xiII, 515.

- Introduction to the Lie Theory of One-Parameter Groups with Applications to the Solution of Differential Equations, E. J. WILCzYNSKI, XVIII, 514.

Combebiac, G. Les Actions à Distance, E. W. Brown, xx, 434.

Coolidge, J. L. The Elements of Non-Euclidean Geometry, J. LIPKA, XVI, 524 . 
Cosserat, E. and F. Théorie des Corps déformables, E. B. WiLson, xIx, 242.

Couturat, L. Les Principes des Mathématiques, avec un Appendice sur la Philosophie des Mathématiques de Kant, J. W. Young, xIv, 147.

— L'Algèbre de la Logique, E. B. WILson, xIv, 175.

- Internaciona matematikal Lexiko en Ido, Germana, Angla, Franca, e Italiana, J. B. SHAw, xvII, 321.

Cox, J. Mechanics, W. H. JACKson, xvI, 542.

Crabtree, H. Theory of Spinning Tops and Gyroscopic Motion, E. W. Brown, xvIr, 209.

Craigo, R. T. See Norris, E. B.

Crathorne, A. R. See Rietz, H. L.

Crelier, L. Systèmes cinématiques, A. EмcH, Xvirr, 316.

Cunningham, A. J. C. Quadratic Partitions, J. C. Morenead, XII, 506.

Czuber, E. Vorlesungen über Differential- und Integral-Rechnung, L. W. Dowling, zweiter Band (zweite Auflage), xv, 392; erster und zweiter Band (dritte Auflage), xx, 418.

- Einführung in die höhere Mathematik, C. L. E. MooRE, xvI, 35.

-Wahrscheinlichkeitsrechnung (zweite Auflage), H. B. PHILlIPs, xx, 429.

Dantscher, V. von. Vorlesungen über die Weierstrass'sche Theorie der irrationalen Zahlen, G. A. Miller, xvI, 83.

Darboux, G. Eloges académiques et Discours, G. A. Miller, XIX, 147.

_ Lecons sur les Systèmes orthogonaux et les Coordonnés curvilignes (deuxième édition), E. J. WILCZYNSKI, XX, 247.

Darwin, G. H. Scientific Papers, E. W. Brown, Vols. I-II, xvI, 73; Vols. III-IV, XviII, 456.

Dassen, C. C. Etude sur les Quantités mathématiques. Grandeurs dirigées. Quaternions, J. W. Young, XII, 128.

Dautheville, S. See Appell, P.

Davaux, E. See Chwolson, O. D.

Davisson, S. C. College Algebra, A. Dresden, xvir, 542.

Denjoy, A. See Baire, R.

Dette, W. Analytische Geometrie der Kegelschnitte, D. D. LEIB, XVII, 40.

Dingeldey, F. Sammlung von Aufgaben zur Anwendung der Differentialund Integralrechnung, erster Teil, E. W. Ponzer, XvIr, 205; zweiter Teil, R. C. Archibald, xx, 482.

Dirichlet, P. L. See Arendt, G.

Doehlemann, K. Geometrische Transformationen, zweiter Teil, V. SNYDER, XVII, 249.

Duhem, P. $\Sigma \omega \zeta \epsilon \iota \nu$ rà $\Phi \iota \iota \nu \delta \mu \epsilon \nu a$, Essai sur la Notion de Théorie physique de Platon à Galilée, E. B. WILson, xvi, 325.

- Etudes sur Léonard de Vinci, D. E. SмIтH, xvIr, 484.

Dumont, E. Arithmétique générale, N. J. LenNes, xvirI, 307, 511; E. DUMONT, XVIII, 508.

Durège, H. Elemente der Theorie der Funktionen einer komplexen veränderlichen Grösse (fünfte Auflage, von L. Maurer), J. PimRPonT, XIV, 191.

Theorie der elliptischen Funktionen (fünfte Auflage, von L. Maurer), J. I. Hutchinson, xv, 132. 
Dziobek, O. Differential- und Integral-Rechnung, G. W. Myers, xIx, 318.

Ebner, F. Technische Infinitesimalrechnung, E. W. PonzER, xx, 158.

Echols, W. H. An Elementary Textbook on the Differential and Integral Calculus, M. W. HASKeLL, XIII, 32.

Einstein, A., and Grossmann, M. Entwurf einer verallgemeinerten Relativitätstheorie und einer Theorie der Gravitation, E. B. WILsoN, $\mathrm{xx}, 273$.

Eisenhart, L. P. Differential Geometry of Curves and Surfaces, G. A. BuIss, xvII, 470; xvIII, 145.

Emch, A. Introduction to Projective Geometry and its Applications, E. B. WILsoN, XII, 132.

Emde, F. See Jahnke, E.

Engel, F. See Grassmann, H.

Enriques, F. Fragen der Elementargeometrie (Deutsche Ausgabe von H. Fleischer), zweiter Teil, H. E. Hawkes, xv, 256.

- Probleme der Wissenschaft. Erster Teil: Wirklichkeit und Logik; zweiter Teil: Die Grundbegriffe der Wissenschaft (Deutsche Uebersetzung von K. Grelling), J. W. YouNG, xIx, 370.

Epstein, P., and Timerding, H. E. Repertorium der höheren Mathematik (zweite Auflage), erster Band, erste Hälfte; zweiter Band, erste Hälfte, C. H. Sisam, XIX, 372 .

Evans, G. W. The Teaching of High School Mathematics, E. B. LytLE, $\mathrm{xx}, 211$.

Faber, G. See Christoffel, E. B.

Fabry, E. Traité de Mathématiques générales à l'Usage des Chimistes, Physiciens, Ingénieurs, et des Elèves des Facultés des Sciences, C. L. E. MOORE, xv, 395.

- Problèmes et Exercices de Mathématiques générales, J. B. SHAw, XVII, 320.

Fagnano, G. C. dei T. di. Opere matematiche (edited by V. Volterra, G. Loria, and D. Gambioli), D. E. SMITH, XIx, 246.

Fazzari, G. Breve Storia della Matematica dai Tempi antichi al medio Evo, D. E. SMItH, xIII, 506.

Ferris, C. E. Elements of Descriptive Geometry, L. I. Hewes, xIII, 142.

Field, P. See Ziwet, A.

Fields, J. C. Theory of the Algebraic Functions of a Complex Variable, J. I. Hutchinson, XIv, 395 .

Fine, H. B. A College Algebra, E. V. Huntington, xIr, 305.

Fine, H. B., and Thompson, H. D. Coordinate Geometry, E. B. Cowler, $\mathrm{XvI}, 314$.

Fischer, P. B. Koordinatensysteme, E. J. WILczynski, xx, 212.

Fischer, V. Vektordifferentiation und Vektorintegration, E. B. WiLson, $\mathrm{XI}, 17$.

Fisher, I. Brief Introduction to the Infinitesimal Calculus, E. L. Dodd, XIII, 512.

— The Nature of Capital and Income, E. B. WILson, xv, 169. The Rate of Interest, E. B. Winson, xv, 169.

Fisher, I., and Brown, H. G. The Purchasing Power of Money, E. B. WiLson, $\mathrm{xx}, 377$.

Fitz-Patrick, J. See Ball, W. W. R. 
Fleck, A., Maennchen, P., and Perron, O. Vermeintliche Beweise des Fermatschen Satzes, J. LIPKA, xVIII, 194.

Fleischer, H. See Enriques, F.

Fleming, J. A. Elektrische Wellen-Telegraphie (Deutsche Ausgabe von E. Aschkinass), E. B. WILson, xv, 143.

Föppl, A. Vorlesungen über technische Mechanik, E. B. WILson, erster und dritter Band (dritte Auflage), XIII, 520; sechster Band, xvII, 548; erster Band (vierte Auflage), xIx, 321.

- See Abraham, M.

Forsyth, A. R. A Treatise on Differential Equations (third edition), E. J. WILCZYNSKI, XII, 130.

- Lehrbuch der Differentialgleichungen (Deutsche Uebersetzung von W. Jacobsthal, zweite Auflage), R. D. CARMICHAEL, XIX, 256.

Fouët, E. A. Leçons élémentaires sur la Théorie des Fonctions analytiques (deuxième édition), G. N. BAUER, XVIII, 30.

Frankland, W. B. Theories of Parallelism, D. E. SмIтH, xvIr, 312.

Fréchet, M. See Borel, E., Heywood, H. B.

Freund, L. See Ball, W. W. R.

Fricke, R. Hauptsätze der Differential- und Integralrechnung (vierte Auflage), H. E. SladGat, XII, 498.

Friedel, G. Leçons de Cristallographie, J. B. SHAw, xvıII, 364.

Gale, A. S. See Smith, P. F.

Galois, E. See Tannery, J.

Gambioli, D. See Fagnano, G. C. dei T. di.

Gans, R. Einführung in die Vektoranalysis mit Anwendung auf die mathematische Physik (zweite Auflage), H. B. PHILlips, xvir, 100.

— Einführung in die Theorie des Magnetismus, E. B. WILsON, xVII, 350.

Gauss, C. F. Werke, Band VII, E. B. WILson, xIII, 521.

Gerland, E. Leibnizens nachgelassene Schriften physikalischen, mechanischen, und technischen Inhalts, F. CAJORI, XIII, 252.

Ghersi, I. Matematica dilettevole e curiosa, R. C. ARCHIBALd, xx, 238.

Gibbs, J. W. Diagrammes et Surfaces thermodynamiques (traduction de G. Roy), W. F. Durand, xI, 446.

- Elementary Principles in Statistical Mechanics, Developed with Especial Reference to the Rational Foundations of Thermodynamics, J. HadAMard, XII, 194.

- Scientific Papers, E. B. WILson, xIII, 250.

Gmeiner, J. A. See Stolz, O.

Goodenough, G. A. See Townsend, E. J.

Goursat, E. A Course in Mathematical Analysis (translated by E. R. Hedrick), W. F. OsGood, XII, 263.

- Cours d'Analyse mathématique, Tome deuxième, W. F. OsGood, xv, 120.

Granville, W. A. Elements of the Differential and Integral Calculus, E. B. VAN VLECK, XII, 181.

- Plane and Spherical Trigonometry and Four-Place Tables of Logarithms, J. WESTLUND, XVI, 381.

- See Smith, P. F.

Grassmann, H. Gesammelte mathematische und physikalische Werke (herausgegeben von F. Engel), zweiter Band, erster und zweiter Teil, E. B. WILson, xIv, 33. 
Projektive Geometrie der Ebene unter Benutzung der Punktrechnung dargestellt, erster Band, L. W. Dowling, xvI, 475.

Gray, G. J. A Bibliography of the Works of Sir Isaac Newton together with a List of Books Illustrating his Works, D. E. SmITH, xv, 188.

Grelling, K. See Enriques, F.

Grimsehl, E. Angewandte Potentialtheorie in elementarer Behandlung, E. B. WILSON, xv, 198.

Grossmann, M. See Einstein, A.

Guichard, M. Sur les Systèmes triplement indéterminés et sur les Systèmes triple-orthogonaux, L. P. EIsENHART, XIII, 23.

Gundelfinger, S. See Hesse, O.

Günther, S. Geschichte der Mathematik, erster Theil, D. E. SMIтн, xv, 245.

Gutzmer, A. Reformvorschläge für den mathematischen und naturwissenschaftlichen Unterricht, zweiter Teil, J. W. A. YounG, xIII, 304.

— Die Tätigkeit der Unterrichtskommission der Gesellschaft Deutscher Naturforscher und Aertze, J. W. A. Young, xv, 261.

Hack, F. Wahrscheinlichkeitsrechnung, G. W. MYERS, xIX, 319.

Halsted, G. B. Rational Geometry, S. C. Davisson, xI, 330. Géométrie rationelle, Traité élémentaire de la Science de l'Espace (traduction française par P. Barbarin), R. C. ARCHIBALD, XIX, 23.

Hancock, E. L. Applied Mechanics for Engineers, E. W. Ponzer, xvIr, 45.

- See Slocum, S. E.

Hancock, H. Lectures on the Calculus of Variations, E. R. HEDRICK, XII, 172.

Haret, S. C. Mécanique sociale, K. Laves, xIx, 32.

Harris, F. Gravitation, E. B. Wilson, xx, 328.

Hauck, G. Vorlesungen über darstellende Geometrie, erster Band, V. SNYDER, $\mathrm{xx}, 253$.

Hawkes, H. E. Advanced Algebra, G. D. OLDs, xII, 405.

- Higher Algebra, J. E. RowE, xx, 209.

Hawkes, H. E., Luby, W. A., and Touton, F. C. First Course in Algebra, J. V. MCKeLVEY, XVIII, 199.

- Second Course in Algebra, J. V. McKelvey, XIX, 29.

Heath, T. L. The Thirteen Books of Euclid's Elements translated from the Text of Heiberg with Introduction and Commentary, D. E. SMIтH, $\mathrm{xv}, 386$.

— Diophantus of Alexandria (second edition), L. E. Dickson, xvirr, 82. The Method of Archimedes Recently Discovered by Heiberg, D. E. SMITH, xIX, 248. Archimedes' Werke (Deutsch von F. Kliem), D. E. SмIтH, xx, 491.

Hedrick, E. R. Calculus of Variations, Encyclopedia Americana, E. R. HEDRICK, XII, 172.

— An Algebra for Secondary Schools, J. Pierpont, xv, 134.

- See Goursat, E.

Hedrick, E. R., and Kellogg, O. D. Applications of the Calculus to $\mathrm{Me}-$ chanics, D. C. GillesPIE, XIX, 148.

Heffter, L., and Koehler, C. Lehrbuch der analytischen Geometrie, erster Band, E. B. CowLEY, XIII, 247.

Heger, R. Analytische Geometrie auf der Kugel, L. W. Dowlıng, xvI, 88. 
Heiberg, J. L. Naturwissenschaften und Mathematik im klassischen Altertum, D. E. Smith, xIx, 84.

Archimedis Opera Omnia, Vol. II, D. E. SмIтH, xx, 489.

See Abhandlungen, Heath, T. L., Tannery, P.

Helmholtz, H. von. Vorlesungen über theoretische Physik (herausgegeben von König und Runge, Krigar-Menzel, und Richarz), E. B. WILsoN, XIII, 112.

Henrici, O., and Turner, G. Vectors and Rotors, with Applications, E. B. WILSON, XI, 17.

Hensel, K. Theorie der algebraischen Zahlen, erster Band, L. E. Dickson, xvII, 23.

Zahlentheorie, L. E. Dickson, xx, 258.

Hering, K. Das 200-jährige Jubiläum der Dampfmaschine, 1706-1906, F. CAJORI, XIV, 453.

Hermite, C. Oeuvres de Charles Hermite (publiées par E. Picard), J. Pierpont, Tome premier, xIII, 182; Tome deuxième, xvI, 370; Tome troisième, XIX, 83.

_Correspondance d'Hermite et de Stieltjes, publiée par B. Baillaud et H. Bourget, Vols. I-II, J. Pierpont, Xv, 504.

Hesse, O. Vorlesungen aus der analytischen Geometrie der geraden Linie, des Punktes und des Kreises in der Ebene (vierte Auflage, von S. Gundelfinger), E. J. WILCZYNSKI, xv, 186.

Hessenberg, G. Transcendenz von $e$ und $\pi$, T. H. GronwalL, xx, 421.

Heywood, H. B., and Fréchet, M. L'Equation de Fredholm et ses Applications à la Physique mathématique, W. R. LoNGLeY, XIX, 236.

Hilbert, D. Grundlagen der Geometrie (dritte Auflage), A. R. Schweitzer, xv, 510; (vierte Auflage), T. H. Gronwall, xx, 325.

- Grundzüge einer allgemeinen Theorie der linearen Integralgleichungen, T. H. Gronwall, xx, 326.

Hilprecht, H. V. Mathematical, Meteorological and Chronological Tablets from the Temple of Nippur, D. E. SMITH, XIII, 392.

Hilton, H. Mathematical Crystallography and the Theory of Groups of Movements, R. P. BAKER, XI, 379.

- An Introduction to the Theory of Groups of Finite Order, A. RANUM, $\mathrm{xv}, 239$.

Hime, H. W. L. Anharmonic Coordinates, J. V. McKelvex, XIX, 416.

Höfler, A. Didaktik des mathematischen Unterrichts, D. E. SмIтH, xIX, 249.

Holton, E. E. Shop Mathematics, C. N. Haskins, xviII, 137, 201, 306; C. F. WARNER, XVIII, 303.

Holzmüller, G. Die Planimetrie für das Gymnasium; Methodisches Lehrbuch der Elementar-Mathematik; Vorbereitende Einführung in die Raumlehre, D. E. SMITH, xII, 458.

Horn, J. Einführung in die Theorie der partiellen Differentialgleichungen, A. R. Crathorne, xix, 254.

Hosmer, G. L. Azimuth, E. B. Wirson, xvirI, 144.

Hovestadt, H. See Killing, W.

Hulburt, L. S. Differential and Integral Calculus, D. D. LEIB, XIX, 197.

Humbert, G. Cours d'Analyse, E. R. HedRICK, XI, 319.

Hun, J. G., and MacInnes, C. R. Elements of Plane and Spherical Trigonometry, C. B. HeNneL, $\mathrm{xx}, 99$. 
Huntington, E. V. The Continuum as a Type of Order; An Exposition of the Modern Theory, O. Veblen, XII, 302.

- The Fundamental Laws of Addition and Multiplication in Elementary Algebra, N. J. LENNES, XVII, 362.

Ignatowski, W. von. Die Vektoranalysis und ihre Anwendung in der theoretischen Physik, H. B. PhIllips, xvIr, 100.

Internationale Mathematische Unterrichtskommission, Berichte und Mitteilungen, Hefte IV-VII, E. W. Ponzer, XIX, 20.

Jackson, C. S., and Milne, R. M. A First Statics, F. L. Griffin, xvi, 142.

Jackson, L. L. See Young, J. W. A.

Jacob, L. Le Calcul mécanique, C. C. Grove, xIx, 30.

Jacobsthal, W. See Forsyth, A. R.

Jahnke, E. Vorlesungen über die Vektorenrechnung, E. B. WILson, xII, 352.

Jahnke, E., and Emde, F. Funktionentafeln mit Formeln und Kurven, E. B. WILSON, XVII, 350 .

James, G. O. Elements of the Kinematics of a Point and the Rational Mechanics of a Particle, K. Laves, XIII, 516.

Jaumann, G. Die Grundlagen der Bewegungslehre, von einem modernen Standpunkte aus, G. W. MYers, XIv, 35.

Jeans, J. H. An Elementary Treatise on Theoretical Mechanics, E. W. Brown, xv, 165.

Johnson, W. W. Differential Calculus, E. W. Ponzer, xvir, 318.

Joly, C. J. A Manual of Quaternions, J. B. SHAw, xr, 548.

Jordan, W. L. Astronomical and Historical Chronology, E. W. Brows, XII, 317.

Jouffret, E. Mélanges de Géométrie à quatre Dimensions, P. FiELd, XIII, 301.

Jouguet, E. Lectures de Mécanique, première Partie, W. R. Longley, xv, 466 ; deuxième Partie, E. B. WILson, xviII, 32.

Junker, F. Repetitorium und Aufgabensammlung, H. E. SLAUGHT, XII, 498.

Kelland, P. See Knott, C. G.

Kellogg, O. D. See Hedrick, E. R.

Kelvin, Lord. Mathematical and Physical Papers. Vols. IV, V, VI, edited by J. Larmor, E. B. WiLson, xx, 431 .

Killing, W., and Hovestadt, H. Handbuch des mathematischen Unterrichts, erster Band, D. D. LeIB, XvII, 258.

King, W. I. Elements of Statistical Method, A. C. LUNN, xIx, 535.

Klaess, P. See Tannery, J.

Klein, F. Ueber eine zeitgemässe Umgestaltung des mathematischen Unterrichts an den höheren Schulen, J. W. A. YounG, xIr, 347.

- Elementarmathematik vom höheren Standpunkte aus, J. W. YounG, XVI, 254.

Klein, F., and Riecke, E. Neue Beiträge zur Frage des mathematischen und physikalischen Unterrichts an den höheren Schulen, J. W. A. YounG, XII, 347.

Klein, F., and Schimmack, R. Vorträge über den mathematischen Unterricht an den höheren Schulen, erster Theil, J. W. A. Young, xv, 511.

Kliem, F. See Heath, T. L.

Kneser, A. Lehrbuch der Variationsrechnung, E. R. HEDRICK, XII, 172. 
- Die Integralgleichungen und ihre Anwendungen in der mathematischen Physik, W. A. HURwITz, XIX, 406.

Knott, C. G. Kelland and Tait's Introduction to Quaternions (third edition), E. B. Wilson, xI, 17.

Koehler, C. See Heffter, L.

Koenigsberger, L. Carl Gustav Jacob Jacobi. Festschrift zur Feier der hundertsten Wiederkehr seines Geburtstages, J. PIerponT, XIr, 261. Hermann von Helmholtz, E. B. Wirson, XIII, 112.

Koepp, G. Illustrierter Spezialkatalog mathematischer Modelle und Apparate, R. C. ARCHIBALD, xx, 244.

Kommerell, V. and K. Spezielle Flächen und Theorie der Strahlensysteme, E. B. Cowlex, XIX, 253.

König, A. See Helmholtz, H. von.

König, J. Einleitung in die allgemeine Theorie der algebraischen Grössen, L. E. Dickson, XIII, 348.

Korn, A. Ueber freie und erzwungene Schwingungen, J. B. SHAw, XIX, 88.

Kowalewski, G. Einführung in die Determinantentheorie, M. BôcHER, XVII, 120.

— Grundzüge der Differential- und Integralrechnung, R. L. BöRGER, XIX, 531 .

- Die komplexen Veränderlichen und ihre Funktionen, A. DRESDEN, xx, 262.

- See Cesàro, E.

Krause, M. Theorie der elliptischen Funktionen (unter Mitwirkung von E. Naetsch), E. B. WrLson, xIx, 427.

Krazer, A. Lehrbuch der Thetafunktionen, J. I. Hutchinson, XI, 375.

- Verhandlungen des dritten internationalen Mathematiker-Kongresses in Heidelberg vom 8 bis 13 August, 1904, H. S. WHITE, xII, 409.

- See Christoffel, E. B.

Krigar-Menzel, O. See Helmholtz, H. von.

Kuenen, J. P. Die Zustandsgleichung der Gase und Flüssigkeiten und die Kontinuitätstheorie, E. B. WrLson, xIV, 339.

Kummer, E. Festschrift zur Feier des 100 Geburtstages Eduard Kummers, L. E. Dickson, XVII, 371.

Lalesco, T. Introduction à la Théorie des Equations intégrales, W. R. LONGLEY, XIX, 236.

Lamb, H. Dynamical Theory of Sound, E. B. Wilson, xix, 260.

Lambert, P. A. Computation and Mensuration, E. W. Ponzer, xiv, 293.

Landau, E. Handbuch der Lehre von der Verteilung der Primzahlen, T. H. Gronwall, xx, 368 .

Lanner, A. Neuere Darstellungen der Grundprobleme der reinen Mathematik im Bereiche der Mittelschule, D. E. SMITH, XIII, 302.

Laplanche, G. de. Etudes sur les Angles imaginaires, J. B. SHAw, xvi, 149.

Larmor, J. Memoir and Scientific Correspondence of the late Sir George Gabriel Stokes, E. W. Brown, XIv, 291. See Kelvin, Lord.

Lattès, S. See Broggi, U., Burali-Forti, C.

Laurent, H. La Géométrie analytique générale, E. B. Cowley, Xv, 363.

- Statistique mathématique, H. L. RIETz, xvI, 322.

Lebesgue, H. Leçons sur l'Intégration et la Recherche des Fonctions Primitives, D. R. Curtiss, XIv, 501. 
Lebon, E. Table de Caractéristiques relatives à la Base 2310 des Facteurs premiers d'un Nombre inférieure à 30,030, G. A. BLIss, xv, 139.

- Henri Poincare, J. W. Young, xvir, 42.

- Gaston Darboux, J. W. Youna, XvII, 258.

—— Emile Picard, J. W. Young, xvirI, 193.

- Paul Appell, J. W. Young, XviIr, 193.

- Gabriel Lippmann, E. B. WrLson, xx, 326.

Lechalas, G. Introduction à la Géométrie Générale, O. VebLen, xI, 439.

Lecornu, L. Dynamique Appliquée, J. B. Staw, xvi, 382.

Lehmer, D. N. Factor Table for the First Ten Millions, L. E. Dickson, XVII, 36.

Leibniz, G. W. See Gerland, E.

Lennes, N. J. See Slaught, H. E., Veblen, O.

Lester, O. C. The Integrals of Mechanics, C. F. CraIG, xvir, 376.

Levy, A. See Sommer, J.

Liebmann, H. Nichteuklidische Geometrie, E. B. CowLEY, XII, 511.

See Bonola, R., Markoff, A. A.

Lietzmann, W., and Trier, V. Wo steckt der Fehler? Trugschlüsse und Schülerfehler, R. C. ARcinibaLd, xx, 238.

Lilienthal, R. von. Vorlesungen über Differentialgeometrie, erster Band, E. J. WILCZYNSKI, XVII, 200.

Lind, B. Ueber das letzte Fermatsche Theorem, J. LIPKA, Xvirr, 194.

Lindelöf, E. Le Calcul des Résidus et ses Applications à la Théorie des Fonctions, W. B. Ford, XII, 134.

Lindemann, F. See Clebsch, A.

Lindemann, F., and L. See Picard, E., Poincaré, H.

Lindt, R. See Abhandlungen.

Loewy, A. Versicherungsmathematik, S. EPsteen, xI, 21.

Loney, S. L. Dynamics of a Particle and of Rigid Bodies, W. R. Longley, XVII, 211.

Longley, W. R. See Smith, P. F.

Lorentz, H. A. The Theory of Electrons and its Applications to the Phenomena of Light and Radiant Heat, E. B. Wirson, xvII, 194.

Loria, G. Il Passato ed il Presente delli principali Teorie geometriche (terza edizione), E. KASNER, xv, 402.

- Vorlesungen über darstellende Geometrie (Deutsche Uebersetzung von F. Schütte), V. SNYDER, erster Teil, xvI, 136; zweiter Teil, xx, 415.

- Spezielle algebraische und tranzendente ebene Kurven (Deutsche Ausgabe von F. Schütte, zweite Auflage), C. L. E. MoorE, xvir, 545.

- Poliedri, Curve e Superficie secondo i Metodi della Geometria descrittiva, V. SNYDER, XX, 415.

- See Fagnano, G. C. dei T. di.

Love, A. E. H. A Treatise on the Mathematical Theory of Elasticity (second edition), F. R. Sharpe, XVI, 90.

— Differential and Integral Calculus, A. Dresden, xIx, 374.

Some Problems of Geodynamics, E. B. Wilson, xx, 432.

Low, D. A. Practical Geometry and Graphics, V. SNYDER, xx, 253.

Luby, W. A. See Hawkes, H. E.

Lukat, M. See Bianchi, L. 
McCormack, T. J. See Mach, E.

MacColl, H. Symbolic Logic and its Applications, E. B. WILson, XIv, 175.

Macfarlane, A. Bibliography of Quaternions and Allied Systems of Mathematics, H. E. HAwKES, XIII, 30.

Mach, E. Space and Geometry in the Light of Physiological, Psychological and Physical Inquiry (translated by T. J. McCormack), C. J. KEYSER, XIII, 197.

MacInnes, C. R. See Hun, J. G.

Maennchen, P. Geheimnisse der Rechenkünstler, D. E. SмIтH, xx, 492.

- See Fleck, A.

Mannoury, G. Methodologisches und Philosophisches zur ElementarMathematik, D. E. SmITH, XIX, 86.

Manville, $O$. Les Découvertes modernes en Physique (deuxième édition), E. B. WILSON, XVI, 92.

Marcolongo, R. See Burali-Forti, C.

Markoff, A. A. Wahrscheinlichkeitsrechnung (zweite Auflage, übersetzt von H. Liebmann), A. C. LuNN, xIX, 535.

Martin, L. A. A Textbook of Mechanics, Vols. I-II, F. L. Griffin, xvi, 142.

Mason, M. See Moore, E. H.

Mathews, G. B. Algebraic Equations, F. CAJorr, xIv, 448.

Maurer, L. See Christoffel, E. B., Durège, H.

Mercer, J. W. The Calculus for Beginners, E. B. Wilson, xx, 30.

Mersereau, S. F. See Breckenridge, W. E.

Meyer, R. See Zeuthen, H. G.

Meyer, W. F. Differential- und Integralrechnung. Zweiter Band: Integralrechnung, V. SNYDER, XII, 133.

- Allgemeine Formen- und Invariantentheorie, erster Band, V. SNYDER, XVI, 437.

- Ueber die Theorie benachbarter Geraden und einen verallgemeinerten Krümmungsbegriff, E. B. CowLEY, XX, 324.

Mills, J. Introduction to Thermodynamics, E. B. WiLson, XIX, 428.

Milne, R. M. See Jackson, C. S.

Minkowski, H. Diophantische Approximationen. Eine Einführung in die Zahlentheorie, L. E. Dickson, xv, 251.

Montessus, R. de. Calcul des Probabilités, E. B. Wilson, xvir, 377.

Moore, C. F. See Breckenridge, W. E.

Moore, E. H., Wilczynski, E. J. and Mason, M. The New Haven Colloquium, G. D. BIRKHOFF, XVII, 414.

Moritz, R. E. College Mathematics Notebook, E. B. Wirson, xx, 327.

- College Engineering Notebook, E. B. WILsoN, xx, 327.

Moulton, F. R. An Introduction to Celestial Mechanics, A. O. Ledschner, XII, 356.

Muir, T. Theory of Determinants; in the Historical Order of Development (second edition), G. A. MILLER, Vol. I, xIII, 244; Vol. II, xVIII, 512.

Müller, C. H. See Abhandlungen.

Müller, Emil. Lehrbuch der darstellenden Geometrie für technische Hochschulen, V. SNYDER, erster Band, xvr, 136; zweiter Band, erster Theil, xx, 253. 
Müller, Eugen. Abriss der Algebra der Logik, L. I. NeIkIRK, xvIII, 515

Müller, F. Führer durch die mathematische Literatur mit besonderer Berücksichtigung der historisch wichtigen Schriften, G. A. Milner, $\mathrm{xv}, 403$.

— Gedenktagebuch für Mathematiker (dritte Auflage), E. W. Ponzer, $\mathrm{xx}, 157$.

Murray, D. A. First Course in Infinitesimal Analysis, W. B. Fite, xI, 442.

- Differential and Integral Calculus, W. B. Carver, xvir, 206.

- Elements of Plane Trigonometry, C. B. Hennel, xx, 156.

Naetsch, E. See Krause, M.

Natorp, P. Die logischen Grundlagen der exakten Wissenschaften, J. W. Young, XIX, 370.

Netto, E. Elementare Algebra, J. H. TANNER, XI, 441.

- Gruppen- und Substitutionentheorie, W. B. FITE, XvI, 33. Die Determinanten, J. B. SHAw, xvII, 547.

Neumann, E. R. Studien über die Methoden von C. Neumann und G. Robin zur Lösung der beiden Randwertaufgaben der Potentialtheorie, O. D. KeLLOGG, XIII, 404.

Nichols, E. W. Analytic Geometry (revised edition), G. H. Scotr, xvI, 491.

Nielsen, N. Handbuch der Theorie der Cylinderfunktionen, F. H. SAFFORD, XIII, 195.

- Handbuch der Theorie der Gammafunktion, V. SNYDER, xIII, 298. Lehrbuch der unendlichen Reihen, J. B. Shaw, xvi, 244.

Norris, E. B., and Craigo, R. T. Advanced Shop Mathematics, P. F. SMITH, $\mathrm{xx}, 428$.

Ocagne, M. d'. Calcul graphique et Nomographie, L. I. Hewes, xv, 127. Le Calcul simplifié, L. I. Hewes, xv, 127.

Orlich, E. Theorie der Wechselströme, E. B. Wilson, xIx, 321.

Osgood, W. F. Lehrbuch der Funktionentheorie, erster Band (erste Auflage), H. S. WHITE, xIII, 398; erster Band (zweite Auflage), E. B. VAN VLECK, $\mathrm{xx}, 532$.

- A First Course in the Differential and Integral Calculus, C. N. HASKINS, xv, 457.

Ostwald, W. Grundriss der Naturphilosophie, E. B. WILson, xvir, 265.

Padoa, A. La Logique déductive dans sa dernière Phase de Développement, J. B. SHAw, xx, 97.

Pareto, V. Manuel d'Economie politique, E. B. Wilson, xvin, 462.

Pascal, E. Calcolo delle Variazioni; Variationsrechnung (Deutsch von A. Schepp), E. R. HEDRICK, xII, 172.

Pasch, M. Grundlagen der Analysis (unter Mitwirkung von C. Thaer), F. W. OWENS, XVI, 213.

Perron, O. See Fleck, A.

Pesloüan, C. L. de. N. H. Abel, sa Vie et son Oeuvre, F. CAJORI, xIIr, 305.

Petit, E. See Richard, P. J.

Picard, E. Sur le Développement de l'Analyse et ses Rapports avec diverses Sciences: Conférences faites en Amérique, E. B. Wilson, xIv, 444 .

— La Science moderne et son Etat actuel, E. B. WILson, xIv, 444. 
- Das Wissen der Gegenwart in Mathematik und Naturwissenschaften (deutsche Ausgabe von F. und L. Lindemann), J. B. SHAw, xx, 424. See Hermite, C.

Picard, E., and Simart, G. Théorie des Fonctions algébriques de deux Variables indépendantes, Tome deuxième, J. I. HuTchrnson, xv, 495.

Pierpont, J. Theory of Functions of Real Variables, Vol. I, G. A. BuIss, XIII, 119; XX, 146.

Pionchon, J. Grandeurs géométriques, J. W. Young, xIr, 127.

- Mathématiques. Principes et Formules de Trigonométrie rectiligne et sphérique, G. W. MYers, xIv, 26.

Planck, M. Das Prinzip der Erhaltung der Energie (zweite Auflage), E. B. WILSON, XVII, 264.

Pockels, F. Lehrbuch der Kristalloptik, E. B. WILson, xvI, 37.

Poincaré, H. Wissenschaft und Hypothese (deutsche Ausgabe von F. und L. Lindemann), E. B. Wrrson, xIr, 187.

- Leçons de Mécanique céleste professées à la Sorbonne, Tome premier; Tome deuxième, première Partie, F. R. Moulton, xv, 258.

— Sechs Vorträge über ausgewählte Gegenstände aus der reinen Mathematik und mathematischen Physik, G. D. BIRKноFF, xvIr, 190.

- Der Wert der Wissenschaft (Uebertragen von E. Weber, zweite Auflage), J. B. SHAw, xIx, 252.

- Calcul des Probabilités (rédaction de A. Quiquet, deuxième édition), R. D. Carmichael, xix, 259.

Prasad, G. Differential Calculus, E. B. WiLson, xIx, 363.

- Integral Calculus, E. B. WILson, xIx, 363.

Quiquet, A. See Poincaré, $H$.

Randall, O. E. Elements of Descriptive Geometry, L. I. Hewes, XIII, 142.

Rangācārya, M. The Ganita-Sāra-Sangraha of Mahāvīācārya, D. E. SMITH, XIx, 310.

Reid, L. W. The Elements of the Theory of Algebraic Numbers, E. B. SKINNER, Xx, 147.

Richard, P. J. Etude sur l'Assurance complémentaire de l'Assurance sur la Vie, C. C. Grove, XIX, 89.

Richard, P. J., and Petit, E. Théorie mathématique des Assurances, E. B. WILson, xvIr, 430.

Richarz, F. Anfangsgründe der Maxwellschen Theorie, E. B. WrLson, XVII, 321.

- See Helmholtz, H. von.

Richter, O. Kreis und Kugel in senkrechter Projektion, für den Unterricht und zum Selbststudium, D. D. LEIB, XvI, 379.

Riecke, E. Beiträge zur Frage des Unterrichts in Physik und Astronomie an den höheren Schulen, J. W. A. YounG, XII, 347.

See Klein, F.

Rietz, H. L., and Crathorne, A. R. College Algebra, J. V. McKenvey, XVII, 262.

Riquier, C. Les Systèmes d'Equations aux Dérivées partielles, E. KASNER, $\mathrm{XIX}, 12$.

Rogers, R. A. P. See Salmon, G.

Romilly, P. W. de. Sur les premiers Principes des Sciences mathématiques, J. B. SHAw, Xvi, 320.

Rost, G. Theorie der Riemann'schen Thetafunction, J. I. Hotchinson, $\mathrm{XI}, 375$. 
Rothe, R. See Auerbach, F.

Roy, G. See Gibbs, J. W.

Royal Society of London. Catalogue of Scientific Papers, 1800-1900. Subject Index, Vol. I, Pure Mathematics, G. A. Miller, xv, 192.

Runge, C. Analytische Geometrie der Ebene, M. Bôcher, xvı, 30. See Helmholtz, H. von.

Russell, B. The Principles of Mathematics, Vol. I, E. B. WILson, xI, 74 Essai sur les Fondements de la Géométrie (traduction par A. Cadenat), E. B. WILson, XI, 74.

- See Whitehead, A. N.

Russell, J. W. An Elementary Treatise on Pure Geometry, O. Veblen, $\mathrm{XIV}, 29$.

Sainte-Laguë, A. Notions de Mathématiques, R. C. ARCHIBALd, xIx, 421.

Salmon, G. Analytic Geometry of Three Dimensions (fifth edition, revised by R. A. P. Rogers), Vol. I, V. SNYDER, XIX, 80.

Saxelby, F. M. A Course in Practical Mathematics, D. E. SмIтH, XII, 458.

Schaefer, C. Einführung in die Maxwellsche Theorie der Elektrizität und des Magnetismus, E. B. WiLson, XvIr, 350.

Schafheitlin, P. Die Theorie der Besselschen Funktionen, A. R. CraTHORNE, XVI, 385.

Scheffers, G. Serret's Lehrbuch der Differential- und Integralrechnung (dritte Auflage), erster und zweiter Band, A. R. CRATHORNE, xv, 140; (dritte Auflage), dritter Band, A. R. CrathorNe, XvI, 377; (vierte und fünfte Auflage), zweiter Band, F. IRwIN, xx, 324.

_ Lehrbuch der Mathematik für Studierende der Naturwissenschaften und der Technik (zweite Auflage), A. R. CRAThoRNe, xIX, 419.

Scheibner, W. Beiträge zur Theorie der linearen Transformationen als Einleitung in die algebraische Invariantentheorie, V. SNYDER, XV, 137.

Schepp, A. See Pascal, E.

Schilling, M. Catalog mathematischer Modelle für höheren mathematischen Unterricht, R. C. ARCHIBALD, xx, 244.

Schimmack, R. See Klein, F.

Schlesinger, L. Vorlesungen über lineare Differentialgleichungen, E. J. WILCZYNSKI, XVI, 483.

Schlömilch, O. Uebungsbuch zum Studium der höheren Analysis, J. Pierpont, xir, 262.

Schmall, C. N. First Course in Analytical Geometry, E. B. CowLeY, xur, 246.

Schoenflies, A. Einführung in die Hauptgesetze der zeichnerischen Darstellungsmethoden, V. SNYDER, Xv, 362.

Schoute, P. H. Mehrdimensionale Geometrie. Zweiter Theil: Die Polytope, W. B. CARVER, xIV, 393.

Schröder, R. Die Anfangsgründe der Differentialrechnung und Integralrechnung, H. E. SlaUGHT, XII, 498.

Schubert, H. Beispiel-Sammlung zur Arithmetik und Algebra (dritte Auflage), G. W. MYers, xIv, 28.

- Auslese aus meiner Unterrichts- und Vorlesungspraxis, E. KASNER, $\mathrm{xv}, 400$.

Schulze, A. The Teaching of Mathematics in Secondary Schools, J. L. CoOLIDGE, XIX, 411. 
Schur, F. Grundlagen der Geometrie, F. W. Owens, xvinr, 309.

Schüssler, R. Orthogonale Axonometrie, V. SNYDER, XII, 361.

Schütte, F. Anfangsgründe der darstellenden Geometrie für Gymnasien, V. SNYDER, XIV, 294.

See Loria, G.

Schwahn, P. Mathematische Theorie der astronomischen Finsternisse, K. LAVES, XIX, 31.

Scott, C. A. Cartesian Plane Geometry. Part 1: Analytical Conics, E. G. BILL, Xv, 507.

Séférian, A. Notice sur le Système des six Coordonnées homogènes d'une Droite et sur les Eléments de la Théorie des Complexes linéaires, V. SNYDER, XVIII, 84.

Séguier, J. A. de. Eléments de la Théorie des Groupes abstraits, L. E. DICKson, XI, 159.

Théorie des Groupes finis. Eléments de la Théorie des Groupes de Substitutions, G. A. MILLER, XIX, 356.

Seliwanoff, D. Lehrbuch der Differenzenrechnung, S. EpsteEn, xI, 25.

Serret, J. A. See Scheffers, G.

Shearman, A. T. The Development of Symbolic Logic; a CriticalHistorical Study of the Logical Calculus, E. B. WILSON, XIV, 175.

Shepard, W. K. Problems in Strength of Materials, E. W. Ponzer, xIv, 452.

Siddons, A. W., and Vassall, A. Practical Measurements, E. W. Ponzer, XVIII, 144.

Simart, G. See Picard, E.

Simon, M. Ueber die Entwicklung der Elementar-Geometrie im XIX. Jahrhundert, D. E. SмITH, XIII, 139.

- Methodik der elementaren Arithmetik in Verbindung mit algebraischer Analysis, D. E. SMITH, XII, 139.

Slaught, H. E., and Lennes, N. J. High School Algebra, E. B. LyTLE, Xv, 357.

- Plane Geometry, F. W. Owens, xvIr, 374. Solid Geometry, F. W. Owens, xvirr, 198.

Slocum, S. E., and Hancock, E. L. Text-book on the Strength of Materials, G. W. Myers, xIv, 37 .

Smith, D. E. Rara Arithmetica (second edition), L. L. JACKson, XvI, 312.

- See Wentworth, G.

Smith, P. F., and Gale, A. S. The Elements of Analytic Geometry, Introduction to Analytic Geometry, O. D. KeLLOGG, XII, 90.

Smith, P. F., and Granville, W. A. Elementary Analysis, J. Westrund, $\mathrm{XIX}, 27$.

Smith, P. F., and Longley, W. R. Theoretical Mechanics, W. D. MACMIILAN, XviII, 84.

Sommer, J. Vorlesungen über Zahlentheorie. Einführung in die Theorie der algebraischen Zahlkörper, G. H. LING, xIv, 145.

- Introduction à la Théorie des Nombres algébriques (French translation by A. Levy), E. B. SkiNNeR, xx, 202.

Sommerville, D. M. Y. Bibliography of Non-Euclidean Geometry, R. C. ARCHIBALD XVIII, 254.

Southall, J. P. C. The Principles and Methods of Geometrical Optics, E. B. WILSON, XIX, 74. 
Stäckel, P. See Borel, E.

Staude, O. Analytische Geometrie des Punktes, der geraden Linie und der Ebene, G. N. BAUER, xIv, 452.

- Analytische Geometrie des Punktpaares, des Kegelschnittes und der Fläche zweiter Ordnung, D. D. LEIB, XVII, 39, 369.

Stokes, G. G. See Larmor, J.

Stolz, O., and Gmeiner, J. A. Einleitung in die Funktionentheorie, erste und zweite Abteilung, O. VeBLEN, XII, 119, 501.

Study, E. Vorlesungen über ausgewählte Gegenstände der Geometrie. Erstes Heft: Ebene analytische Kurven und zu ihnen gehörige Abbildungen, A. EMCH, xIx, 15; zweites Heft: Konforme Abbildung einfach-zusammenhängender Bereiche (herausgegeben unter Mitwirkung von W. Blaschke), A. EмcH, xx, 493.

Sturm, R. Die Lehre von den geometrischen Verwandtschaften, V. SNYDER, erster Band, $\mathrm{xv}, 135$; zweiter Band, $\mathrm{xv}, 252$; dritter Band, XVI, 250; vierter Band, XVII, 150.

- Maxima und Minima in der elementaren Geometrie, J. V. McKeLver, $\mathrm{xx}, 427$.

Stuyvaert, M. Cinq Etudes de Géométrie analytique, E. G. BiLl, xvir, 479.

Sylvester, J. J. Collected Mathematical Papers (edited by H. F. Baker), L. E. Dickson, Vols. I-II, xv, 232; Vol. III, xvII, 254.

Tait, P. G. See Knott, C. G.

Tanner, J. H. Elementary Algebra, J. Pierpont, xI, 444.

Tanner, J. H., and Allen, J. A Brief Course in Analytic Geometry, C. B. HENNEL, XX, 156.

Tannery, J. Introduction à la Theorie des Fonctions d'une Variable, Tome premier, L. E. Dickson, XI, 557.

—_ Leçons d'Algèbre et d'Analyse à l'Usage des Elèves des Classes de Mathématiques spéciales, Tome premier, F. CAJoRr, xIv, 21; Tome second, G. W. MYERs, xIv, 24.

- Manuscrits de Evariste Galois, L. E. Dickson, xv, 249.

Elemente der Mathematik (deutsche Ausgabe von P. Klaess), J. B. SHAW, XVII, 367.

Tannery, P. Mémoires scientifiques (publiés par J. L. Heiberg et H. G. Zeuthen), Tome premier: Sciences exactes dans l'Antiquité, D. E. SMITH, XIX, 367.

Tchebychef, P. L. Theory of Congruences, A. Markoff, XI, 337

Teixeira, F. G. Tratado de las Curvas especiales notables, C. H. Sisam, XIII, 249.

Thaer, C. See Pasch, M.

Thiele, T. N. Interpolationsrechnung, H. L. Rietz, xvir, 372.

Thomae, J. Sammlung von Formeln und Sätzen aus dem Gebiete der elliptischen Functionen, nebst Anwendungen, H. E. SlaUGHT, XII, 498.

- Vorlesungen über bestimmte Integrale und die Fourier'schen Reihen, J. B. SHaw, xvi, 150.

Thompson, H. D. See Fine, H. B.

Thompson, S. P. Petrus Peregrinus de Maricourt and his Epistola de Magnete, F. CAJORI, XIV, 295.

Timerding, H. E. Geometrie der Kräfte, W. R. Longley, xvi, 493.

— Theorie der Kräftepläne, J. B. SHAW, xvIr, 547. 
- Die Mathematik in den physikalischen Lehrbüchern, E. W. Ponzer, XVIII, 142.

- See Epstein, P.

Touton, F. C. See Hawkes, H. E.

Townsend, E. J., and Goodenough, G. A. First Course in Calculus, N. J. LENNES, XIX, 316.

- Essentials of Calculus, N. J. Lennes, xIx, 316.

Treutlein, P. See Wiener, $\mathrm{H}$.

Trier, V. See Lietzmann, W.

Tripos, Cambridge University Mathematical, 1906, V. SNYDER, XIII, 131.

Tropfke, J. Geschichte der Elementar-Mathematik in systematischer Darstellung, J. W. A. Young, XII, 138.

Turner, G. See Henrici, O.

Vahlen, K. T. Abstrakte Geometrie, O. Veblen, xII, 505.

Konstruktionen und Approximationen, E. W. Ponzer, xIX, 92.

Van Orstrand, C. E. See Becker, G. F.

Van Vleck, E. B., White, H. S., and Woods, F. S. The Boston Colloquium Lectures on Mathematics, J. I. HuTchinson, XIII, 85.

Vassall, A. See Siddons, A. W.

Veblen, O., and Lennes, N. J. Introduction to Infinitesimal Analysis. Functions of One Real Variable, J. PIERPont, xv, 133.

Veblen, O., and Young, J. W. Projective Geometry, Vol. I, J. L. Coolrdag, xvIII, 70.

Verhandlungen der Breslauer Naturforscher-Versammlung über den naturwissenschaftlichen und mathematischen Unterricht an den höheren Schulen, J. W. A. YounG, xII, 347.

Vessiot, E. Leçons de Géométrie supérieure, C. L. E. Moore, XIII, 509.

Vivanti, G. Funzioni poliedriche e modulari, J. I. HUTCHINson, xIv, 144; (French translation by A. Cahen), G. A. Miller, XIX, 534 .

- Esercizi di Analisí infinitesimale, R. C. ARCHIBALd, xx, 482.

Vogt, W. Synthetische Theorie der Cliffordschen Parallelen und der linearen Linienörter des elliptischen Raumes, E. B. CowLEY, XVII, 315.

Voigt, A. Theorie der Zahlenreihen und der Reihengleichungen, R. D. CARMICHAEL, XIX, 203.

Volkmann, P. Erkenntnistheoretische Grundzüge der Naturwissenschaften (zweite Auflage), J. W. Young, XIX, 370.

Volterra, V. Leçons sur les Equations intégrales et les Equations intégrodiffèrentielles, J. WESTLUND, xx, 259.

- See Fagnano, G. C. dei T. di.

Voss, A. Ueber das Wesen der Mathematik (erste Ausgabe), F. CAJORI, $\mathrm{XV}, 405$.

Walker, B. M. On the Resolution of Higher Singularities of Algcbraic Curves into Ordinary Nodes, H. S. WHITE, xIv, 336.

Wangerin, A. Theorie des Potentials und der Kugelfunktionen, erster Band, J. B. SHAw, Xvi, 492.

Weber, E. See Poincaré, H.

Weber, H. Die partiellen Differentialgleichungen der mathematischen Physik (fünfte Auflage), erster Band, O. D. KeLloGg, xvIIr, 87; zweiter Band, J. B. SHAW, XIX, 482.

- Festschrift Heinrich Weber zu seinem siebzigsten Geburtstag am 5 März 1912, R. C. ARCHIBALd, XX, 152. 
Weber, H., and Wellstein, J. Encyklopädie der Elementar-Mathematik (erste Auflage), zweiter und dritter Band, H. S. WHITE, XIv, 499; (dritte Auflage), erster Band, F. W. OwENs, XvII, 546; (zweite Auflage), dritter Band, J. B. SHAw, xIX, 87, 422.

Webster, A. G. The Dynamics of Particles and Rigid, Elastic, and Fluid Bodies (second edition), W. R. LONGLEY, xx, 159.

Weitzenböck, R. Komplex-Symbolik, C. L. E. MooRE, xvIr, 368.

Wellstein, J. See Weber, H.

Wentworth, G., and Smith, D. E. Complete Arithmetic, G. H. Scotr, XVI, 491.

Weyl, H. Die Idee der Riemannschen Fläche, F. R. Moduton, xx, 384.

White, H. S. See Van Vleck, E. B.

White, W. F. A Scrap-Book of Elementary Mathematics, D. E. SMIтH, $\mathrm{xv}, 190$.

Whitehead, A. N. The Axioms of Descriptive Geometry, F. W. Owens, $\mathrm{xv}, 465$.

Whitehead, A. N., and Russell, B. Principia Mathematica, Vol. I, J. B. SHAW, XVIII, 386.

Whittaker, E. T. A Treatise on the Analytical Dynamics of Particles and Rigid Bodies, E. B. WILson, XII, 451.

- A History of the Theories of Aether and Electricity from the Age of Descartes to the Close of the Nineteenth Century, E. B. WILson, $\mathrm{xIX}, 423$.

Wieleitner, H. Spezielle ebene Kurven, E. G. BILL, XvI, 534.

Wien, W. Ueber Elektronen (zweite Auflage), E. B. WILson, xvir, 194.

Wiener, H., and Treutlein, P. Verzeichnis von $H$. Wieners und P. Treutleins Sammlung mathematischer Modelle für Hochschulen, höhere Lehranstalten und technische Fachschulen. Zweite Ausgabe, R. C. ARCHIBALD, Xx, 244.

- Abhandlungen zur Sammlung mathematischer Modelle, R. C. ARCHIBALD, XX, 244.

Wilczynski, E. J. Projective Differential Geometry of Curves and Ruled Surfaces, V. SNYDER, XIII, 190, 362.

- See Moore, E. H.

Willis, H. G. Elementary Modern Geometry. Part 1: Experimental and Theoretical, Triangles and Parallels, V. SNYDER, xII, 263.

Wilson, E. B. Advanced Calculus, W. E. ByerLY, XIX, 360.

Wilson, V. T. Descriptive Geometry, V. SNYDER, XvI, 136.

Woods, F. S. See Van Vleck, E. B.

Wright, J. E. Invariants of Quadratic Differential Forms, L. P. ErsenHART, XVII, 140.

Young, G. C. See Young, W. H.

Young, J. W. A. Monographs on Topics of Modern Mathematics Relevant to the Elementary Field, R. D. Carmichase, xx, 207.

Young, J. W. A., and Jackson, L. L. Elementary Algebra, E. B. LxtLE, xvI, 215.

Young, J. W. Lectures on Fundamental Concepts of Algebra and Geometry, E. B. LYTLE, XVIII, 362.

- See Veblen, 0.

Young, W. H. The Fundamental Theorems of the Differential Calculus, N. J. LENNES, XVII, 488. 
Young, W. H., and G. C. The Theory of Sets of Points, N. J. Lennes, XVIII, 24.

Zeuthen, H. G. Geschichte der Mathematik in XVI. und XVII. Jahrhundert (deutsche Ausgabe von R. Meyer), D. E. SmitH, XI, 554. See Tannery, $\mathbf{P}$.

Ziwet, A., and Field, P. Introduction to Analytical Mechanics, K. LAves, $\mathrm{xx}, 37$.

Zoretti, L. Leçons sur le Prolongement analytique, F. IrwIN, xx, 321. 